\title{
Stress fiber growth and remodeling determines cellular morphomechanics under uniaxial cyclic stretch
}

\author{
Aritra Chatterjee $^{1} \cdot$ Paturu Kondaiah $^{2} \cdot$ Namrata Gundiah $^{1,3}$ (1) \\ Received: 14 October 2020 / Accepted: 17 December 2021 \\ (c) The Author(s), under exclusive licence to Springer-Verlag GmbH Germany, part of Springer Nature 2021
}

\begin{abstract}
Stress fibers in the cytoskeleton are essential in maintaining cellular shape and influence cellular adhesion and migration. Cyclic uniaxial stretching results in cellular reorientation orthogonal to the applied stretch direction. The mechanistic cues underlying changes to cellular form and function to stretch stimuli are currently underexplored. We show stretch-induced stress fiber lengthening, their realignment, and increased cortical actin in NIH 3 T3 fibroblasts stretched over varied amplitudes and durations. Higher amounts of actin and stress fiber alignment were accompanied with an increase in the effective elastic modulus of cells. Microtubules did not contribute to the measured stiffness or reorientation response but were essential to the nuclear reorientation. We used a phenomenological growth and remodeling law, based on the experimental data, to model stress fiber elongation and reorientation dynamics based on a nonlinear, orthotropic, fiber-reinforced continuum representation of the cell. The model predicts the changes observed fibroblast morphology and increased cellular stiffness under uniaxial cyclic stretch which agrees with experimental results. Such studies are important in exploring the differences underlying mechanotransduction and cellular contractility under stretch.
\end{abstract}

Keywords Nonlinear fiber-reinforced continuum $\cdot$ Effective modulus $\cdot$ Cytoskeleton $\cdot$ Nuclear orientation $\cdot$ Growth and remodeling

\section{Introduction}

D'Arcy Thompson's influential book, titled “On Growth and Form," suggested the importance of physical cues in producing a rich array of biological patterns in nature (Thompson 1917). This work helped spur several studies that seek to understand the design principles underlying biological form and function. Growth and remodeling is a powerful paradigm, frequently referred to as morphometrics, which aims to link the diversity of shapes in living systems to their underlying function. The mechanistic description of growth is of potential interest in our understanding of development

Namrata Gundiah

namrata@iisc.ac.in; ngundiah@gmail.com

1 Centre for BioSystems Science and Engineering, Indian Institute of Science, Bangalore, Karnataka 560012, India

2 Department of Molecular Reproduction, Development and Genetics, Indian Institute of Science, Bangalore, Karnataka 560012, India

3 Department of Mechanical Engineering, Indian Institute of Science, Bangalore, Karnataka 560012, India processes, including morphogenesis and homeostasis, pathological disorders, and in the progression of diseases like fibrosis and aneurysms. Continuum mechanics-based approaches to address biological growth and remodeling suggest an intimate relationship between the cellular level mechanobiology and the underlying tissue properties (Humphrey and Rajagopal 2002).

Adherent fibroblasts in tissues, such as arteries and lungs, undergo cyclic stretch and respond to changes in their mechanical milieu through a complex interplay between the cytoskeletal components and integrin-mediated pathways to influence cell contractility and secretion of extracellular matrix constituents (Robertson and Watton 2013; Hsu et al. 2010). Cell-driven biochemical processes result in gain or loss in mass and induce remodeling of the underlying material properties, such as stiffness and anisotropy, that result in non-uniform changes to the structural form and function over time. How do mechanosensing processes influence cellular growth and remodeling under stretch? Stretch-induced reorientation of cells involves both passive mechanical response to cyclic substrate deformation, and dynamic changes to the cytoskeleton (Livne et al. 2014). Contractile 
stress fibers (SF's), comprised primarily of actin and myosin II, are essential in the development of intracellular stresses which are transmitted through focal adhesion (FA) complexes, and depend on the activity of RhoA, active Rac1, or Cdc42 (Balaban et al. 2001; Cirka et al. 2016; Perrault et al. 2015). Cyclic uniaxial stretching of fibroblasts, seeded on isotropic elastomeric substrates, leads to cell reorientation from random to a near-perpendicular angle with respect to the loading direction (Buck 1980; Wang et al. 2001). Vascular smooth muscle cells show an initial rapid growth and reinforcement followed by disassembly of SF aligned in the direction of stretch; SF's subsequently rearrange in an orthogonal direction (Hayakawa et al. 2001). The associated actin reorientation under stretch is accompanied with an increase in FAK signaling, MAP kinases, and a corresponding dynamic increase in the size of FA complexes (Hsu et al. 2010; Chen et al. 2013; Hoffman et al. 2017). Application of uniaxial cyclic stretch results in activation of p38 MAP kinase and induces actin remodeling (Hoffman et al. 2017). Higher strain rates cause rapid disassembly of SF's along the direction of deformation (Nekouzadeh et al. 2008). The application of cyclic stretch also influences cell spreading on soft substrates. SF formation is known to persist up to four hours after stretching and is related to temporal changes in the movement of MRTF-A and YAP from the cytoplasm to the nucleus (Greiner et al. 2013; Cui et al. 2015).

Stress fibers are exquisitely sensitive to changes in the mechanical environment, and are essential in the regulation of cell polarization and transmission of tensional force from the FA to the cell (Goldyn et al. 2009). Cell spreading, dependent on the stiffness of the underlying substrate, results in a biphasic cellular orientation caused by de novo formation of ventrally oriented actin in the cell. Jungbauer and colleagues (2008) reported that the characteristic time for cellular reorientation decreased with an increase in the applied stretch frequency $(<1 \mathrm{~Hz})$, and remained constant for stretch frequencies higher than $1 \mathrm{~Hz}$. Cell alignment under cyclic uniaxial stretch is hypothesized to be an avoidance reaction to stretch which is facilitated via cell-substrate interactions and their links to the cytoskeleton (Jungbauer et al. 2008). Mean cellular orientation after stretch contributes to the tensional homeostasis and helps maintain an optimal internal stress (Kaunas et al. 2005). More recently, Chen et al. showed that traction boundary conditions are crucial in the determination of cellular orientation under stretch (Chen et al. 2018). The precise mechanistic cues in cellular mechanosensitivity to dynamical environments, and links to SF growth and cell stiffness changes, however, remain a fundamental open question.

The goals of this study are to investigate the morphomechanical changes in fibroblasts due to SF dynamics under uniaxial cyclic stretch, and relate their growth and remodeling to the cellular reorientation response using a continuum mechanics-based nonlinear elasticity framework. We used a custom fabricated stretcher to subject fibroblasts to uniaxial cyclic stretch for different amplitudes over varied time periods. We show that the lengthening and realignment of SF primarily contributes to the cellular remodeling response under cyclic stretch. We model the cell using a nonlinear, hyperelastic, fiber-reinforced material description which includes two families of SF that grow and reorient under uniaxial cyclic stretch. The measured changes in cell stiffness in response to the stretch amplitude and time duration due to SF reorientation are well captured using the model. We report an increase in the cortical thickness of actin under the application of uniaxial cyclic stretch that may contribute to the measured mechanical properties. We also show that microtubules do not contribute to the measured stiffness or reorientation response but were essential in the nuclear reorientation. These results show the specific contributions of SF growth in the elongation and orientation response of cells under cyclic stretch that have not been shown earlier.

\section{Results}

\subsection{Cyclic stretch-induced changes in the cell and nuclear morphologies}

Uniaxial cyclic stretch experiments were performed on NIH 3T3 fibroblasts seeded on thin flexible elastomeric membranes using a custom stretching device (Fig. 1). The device includes a bioreactor, and uses a novel clamping system that allows stretching of thin, cell-seeded membranes based on user-defined stretch amplitudes and frequencies over extended durations. The device with bioreactor was placed within a humidified, temperature-controlled incubator to maintain sterility during experimentation (Fig. 1a, b). Cell-seeded constructs were stretched uniaxially at $5 \%$ and $10 \%$ amplitude at a frequency of $1 \mathrm{~Hz}$ for 3 and $6 \mathrm{~h}$. Confocal images of fibroblasts stretched at $10 \%$ amplitude for $6 \mathrm{~h}$ (A10T6) show distinct changes in the overall cellular morphology (Supplementary Fig. S1). Cells were significantly elongated and elliptical in a direction perpendicular to the applied stretch direction as compared to unstretched controls. The cytoskeletal and nuclear orientations were clearly different at higher amplitude and over longer stretching durations (A10T6) (Fig. 2a, b).

A total of $\sim 30$ cells were included in each set and the data for individual cells were averaged to compute an overall SF angular and length distribution for each group of cells subjected to different amplitudes and durations of cyclic stretch (Table 1). Results show SF realignment in a direction near perpendicular to the direction of applied cyclic stretch (Fig. 2c). SF orientations changed from relatively random orientations in unstretched cells to a unimodal distribution 
(a)

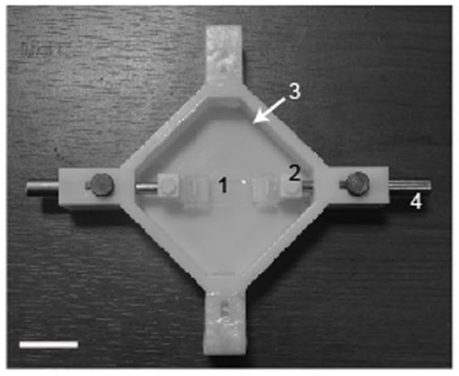

(1) Elastomer Sample

(4) Actuator Arms (b)

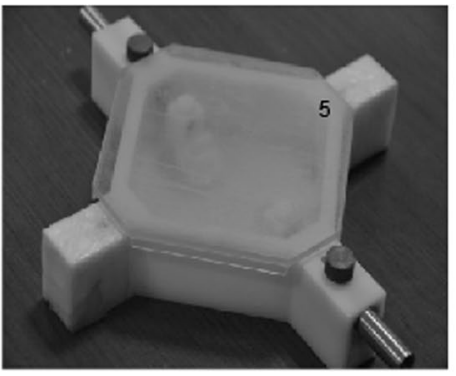

(2) Clamps (3) 3-d printed Bioreactor

(5) Semi-transparent Lid (c)

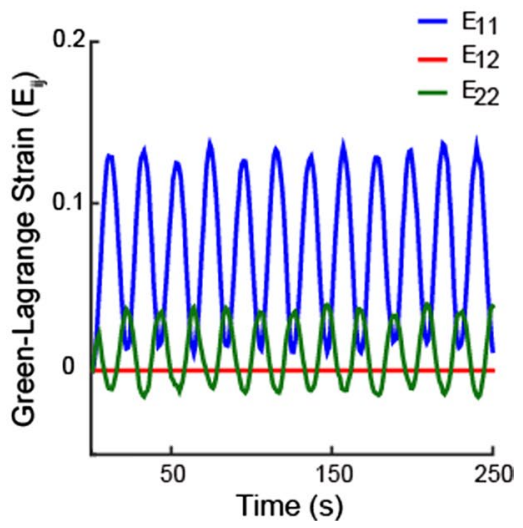

Fig. $1 \mathbf{a}$ and $\mathbf{b}$ Custom designed bioreactor used in the study. A thin PDMS membrane was held using custom designed clamps in the 3D printed bioreactor that may be subjected to uniaxial or biaxial stretch-

orthogonal to the stretch direction due to uniaxial stretch. Because unstretched cells demonstrate random orientations, the standard errors in the measured angular orientation distributions for the control unstretched group are much larger than those for aligned cells obtained at higher amplitudes and cyclic stretch durations (Table 1). Application of cyclic stretch alters the cellular and nuclear morphometrics. To quantify these differences, we segmented and fitted an ellipse to each of the individual cell and nuclear images using ImageJ (NIH) software (Goldyn et al. 2009). The aspect ratio was calculated as a ratio of the major axis to the minor axis of the fitted ellipse. Angles were measured relative to the uniaxial stretch direction. We observe significant differences in the cellular and nuclear aspect ratios ( $p<0.01, p<0.05$, respectively) between cyclically stretched cells (A10T6) and unstretched controls (Fig. 2d). The angular nuclear orientations under stretch were also perpendicular to the applied stretch direction (Fig. 2e).

\subsection{Role of stress fibers in the cellular reorientation and stiffness changes under cyclic stretch}

We used cytoskeletal inhibitors to delineate the individual contributions of SF's and microtubules under cyclic stretch. Cells were treated with cytochalasin-D, to inhibit actin polymerization, and nocodazole (Barreto et al. 2013), to disrupt microtubules, and the constructs stretched uniaxially as described earlier. Submaximal concentrations of the cytoskeletal disruptors were used to prevent cellular detachment from the basement membrane during the application of cyclic stretch or for the AFM indentation experiments (Barreto et al. 2013). Stretching experiments clearly demonstrate that the inhibition of microtubule polymerization did not abrogate the reorientation response of cells; in contrast, inhibition of actin led to a complete loss in the reorientation ing. c Green-Lagrange strains under uniaxial cyclic strain (15\% amplitude) were quantified using particle tracking algorithm based on markers located on the PDMS substrate

response of cells. Both actin and microtubule depolymerizations significantly abrogated the nuclear reorientation under cyclic stretch. Cytochalasin-D treated cells showed $\sim 7^{\circ}$ change in nuclear orientation in the A10T6 group as compared to nocodazole treated cells that did not change under stretch (Fig. 2e). These results show conclusively the primary contribution of actin to the cellular reorientation responses as compared to microtubules that are essential to the nuclear reorientation.

We quantified the differences in cellular stiffnesses after stretch using an atomic force microscope (AFM). Individual cells were indented with a spherical indenter and the modulus was calculated using a thin layer, finite thickness, Hertz model (Kulkarni et al. 2018; Darling et al. 2007; Dimitradis et al. 2002) (Supplementary Information 1). Fibroblasts stretched at $10 \%$ for $6 \mathrm{~h}$ were indented in the presence or absence of cytoskeletal inhibitors, and the corresponding cell stiffnesses were compared with unstretched controls. Our results show that cyclically stretched cells had significantly higher stiffness as compared to unstretched controls ( $p<0.05$; Fig. 2f). There was no significant change in the effective elastic modulus for cytochalasin-D treated cells under stretch. In contrast, nocodazole treated cells showed a significant increase in cell stiffness following stretch $(p<0.01)$. Depolymerization of microtubules did not affect the stretch-induced increased cell stiffness.

We quantified the SF orientations using confocal images of cells stained for actin (Fig. 3a) (Schriefl et al. 2013; Kulkarni et al. 2018). Images were binarized (Fig. 3b), and the SF angular orientations relative to the stretch direction were quantified based on spatial frequencies computed in Fourier space (Fig. 3c, d) using a custom MATLAB code (see Sect. 4.5). The SF lengths were quantified using Hough transform of the confocal images (Fig. 4a, Supplementary Table 1). This method allows us to map any arbitrary straight 
Fig. 2 a and $\mathbf{b}$ Confocal images of unstretched control, and uniaxially stretched fibroblasts at $10 \%$ amplitude for $6 \mathrm{~h}$ stained for F-actin (red), microtubule (green), and DAPI nucleus (blue). Scale bar represents $50 \mu \mathrm{m}$. $\mathbf{c}$ and $\mathbf{d}$ Distribution of SF orientations subjected to uniaxial cyclic stretch $(1 \mathrm{~Hz})$, 5 and $10 \%$ stretch amplitude for $6 \mathrm{~h}$. $(n=33,32$ and 35 cells in control, A5T6 and A10T6 groups, respectively). d Cyclic stretch-induced fibroblast cell and nuclear aspect ratio increased under uniaxial cyclic stretch for control $(n=33)$ and A10T6 $(n=35)$, respectively. e Changes in the angular orientation of nucleus (Mean \pm SEM) under uniaxial cyclic stretch $(1 \mathrm{~Hz})$ with/without cytoskeletal inhibitors compared under 5 and $10 \%$ stretch amplitude for $6 \mathrm{~h}$ for control $(n=33)$, A5T6 $(n=32)$ and A10T6 $(n=35)$ groups, respectively. $\mathbf{f}$ The elastic cell modulus (Mean \pm SEM) for cells subjected to cyclic stretch with/without cytoskeletal disruptors shown for control ( $n=25$, in each group) and stretched cells (A10T6; $n=25$, for each group). Significant differences are indicated for $p<0.01(* *)$ and $p<0.05(*)$; n.s represents not significant (a)
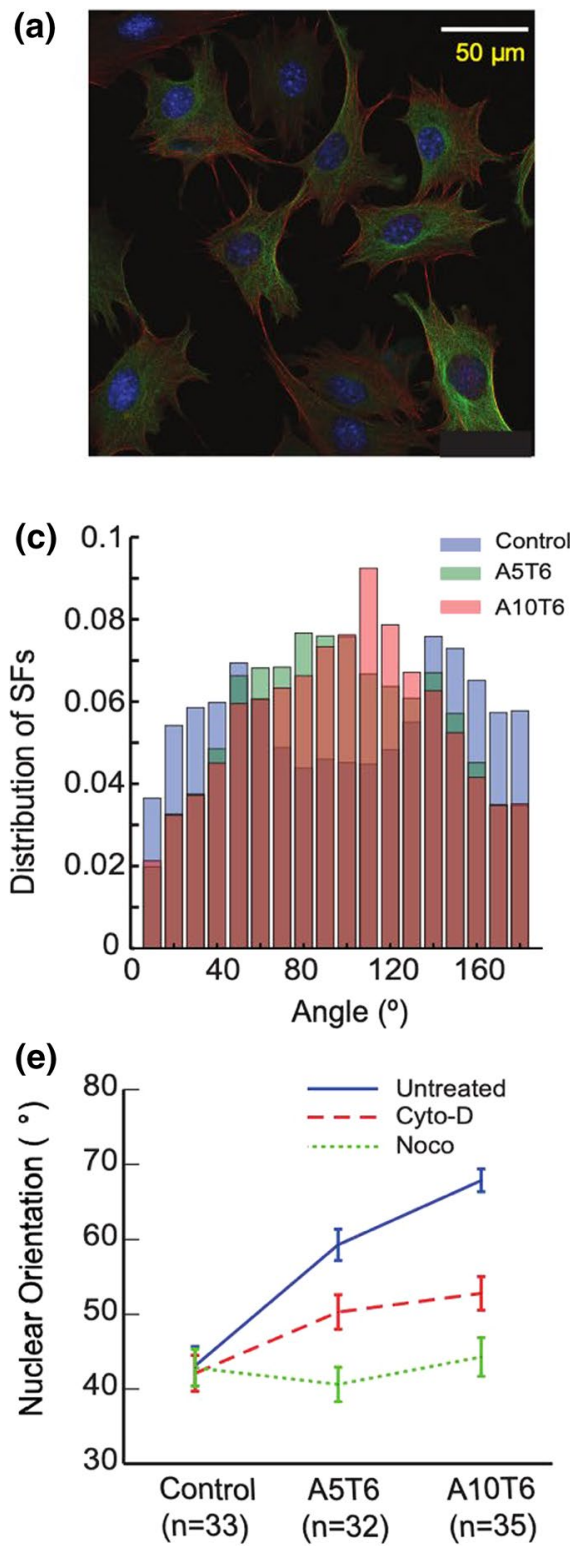

(b)
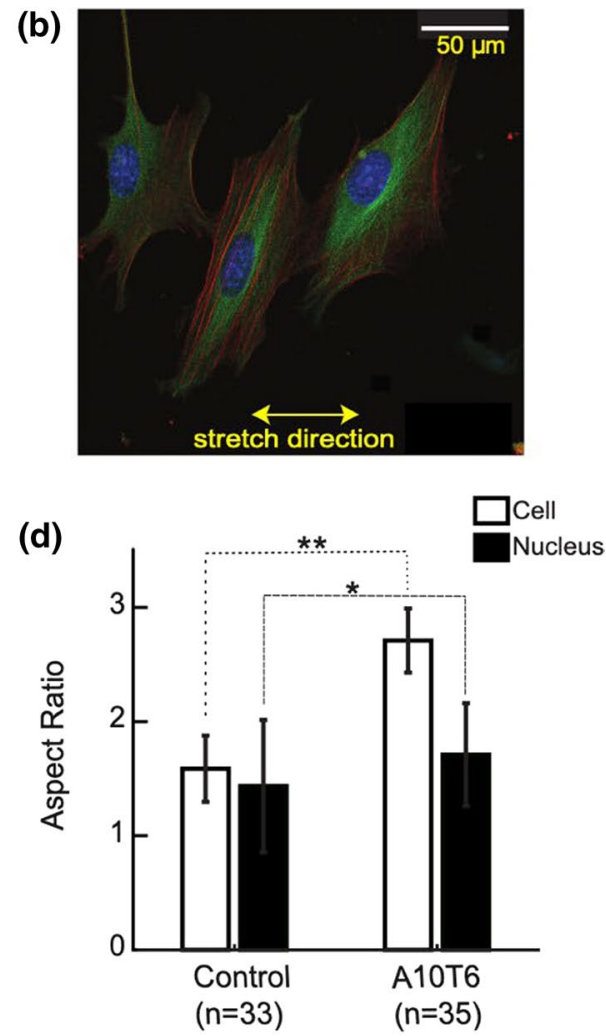

(f)

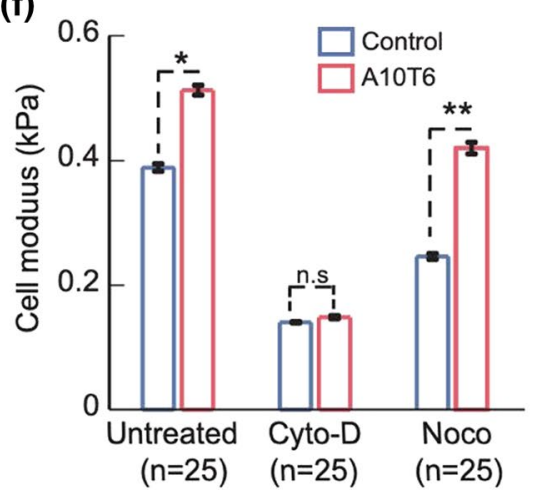

Table 1 Distribution of SF angular orientations and lengths (Mean \pm SEM) for fibroblasts subjected to uniaxial cyclic stretch (5 and $10 \%$ amplitude, stretch frequency $1 \mathrm{~Hz}$ )

\begin{tabular}{lll}
\hline & SF angles $\left(^{\circ}\right)($ Mean \pm SEM) & $\begin{array}{l}\text { SF length }(\mu \mathrm{m}) \\
(\text { Mean } \pm \text { SEM })\end{array}$ \\
\hline Control & $43.6 \pm 4.9$ and $150.9 \pm 7.9$ & $24.22 \pm 2.25$ \\
A5T3 & $51.8 \pm 5.3$ and $148.9 \pm 4.9$ & $33.71 \pm 2.18$ \\
A5T6 & $76.2 \pm 7.5$ and $127.5 \pm 5.5$ & $35.33 \pm 1.5$ \\
A10T3 & $72.7 \pm 7.6$ and $115.2 \pm 7.3$ & $37.19 \pm 2.44$ \\
A10T6 & $79.9 \pm 2.8$ and $99.2 \pm 1.4$ & $44.52 \pm 1.76$ \\
\hline
\end{tabular}

line in the $x-y$ plane to a single point in the Hough parameter space that is defined using the angle of the straight line with the $X$-axis $(\theta)$, and its algebraic distance from the origin $(\rho)$.
Restricting $\theta$ within the interval $(0, \pi)$ ensures uniqueness of the line parameters in Hough space. The binarized images of the cell (Fig. 4b) were used to segment the SF's, and the edge points were stored (Fig. 4c) as demonstrated for a small representative volume element (RVE) of $30 \mu \mathrm{m} \times 30 \mu \mathrm{m}$ highlighted in the figure for clarity. Data from coordinates corresponding to all points in the accumulator were used to compute the SF lengths (Fig. 4d). Additional details are provided in Methods (Sect. 4.6) and in the Supplementary Fig. S2.

We measured the F-actin fluorescence intensity at the cell boundaries and calculated the peak intensity values and full width at half maximum (FWHM) values corresponding to the intensity distribution for each cell. These metrics were used to quantify possible contributions of the cortical actin to the overall increase in cell stiffness 
Fig. 3 a Confocal images show an individual NIH 3T3 fibroblast cell, stained with phalloidin to visualize SF and DAPI to identify the nucleus. Scale bar represents $50 \mu \mathrm{m}$. b Corresponding binarized image of the confocal image is shown. c The computed Fourier power spectrum was obtained using the binary image. $\mathbf{d}$ Distribution of SF's in the cell was calculated using their spatial frequency distributions (a)

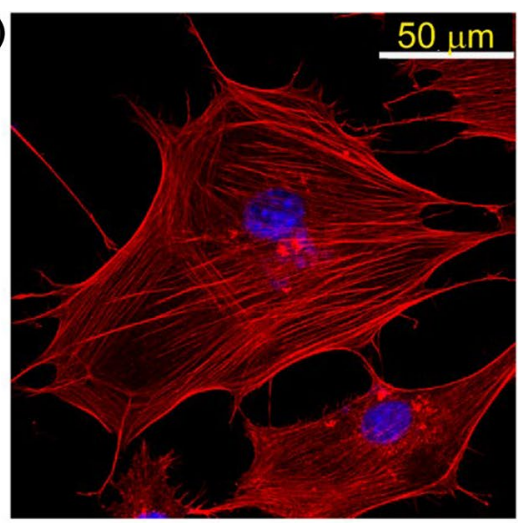

(c)

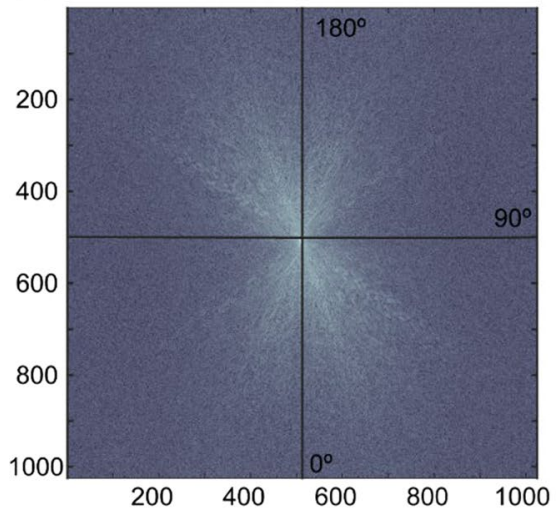

(b)

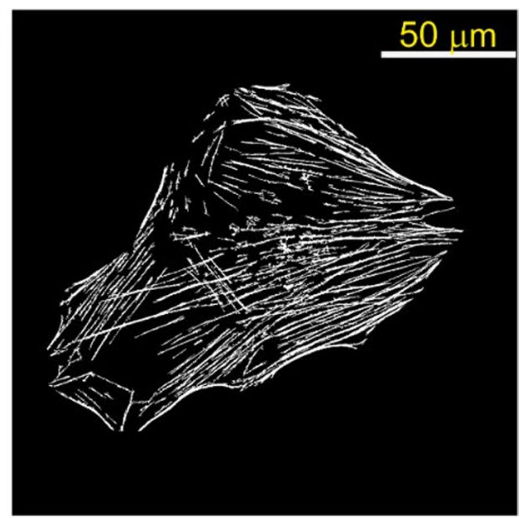

(d)

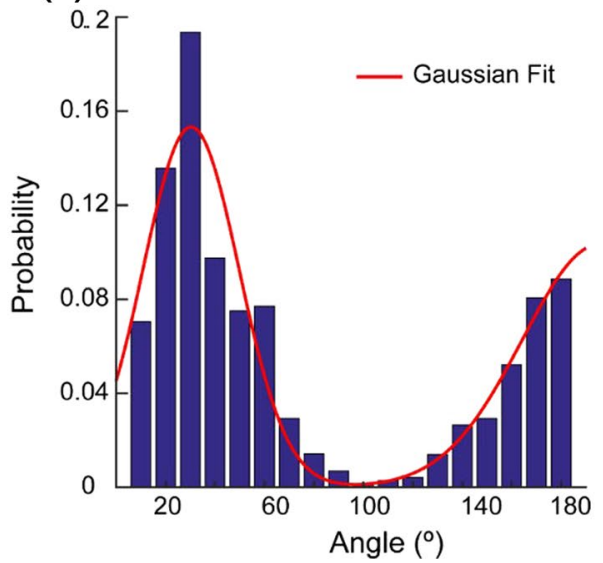

due to stretching (Fig. 5a, b). We used image segmentation techniques to subtract the intracellular and extracellular background intensities from the cellular boundary. The intensity distributions obtained at the cell edges (Fig. 5c) were fit to a Gaussian distribution function to calculate the peak position and intensities (Clark et al. 2013). FWHM values corresponding to the intensity distribution for each cell and the peak intensity values were used to characterize the thickness of the cortical actin for the cell (Fig. 5d, e). FWHM was $40 \%$ higher for cyclically stretched (A10T6) cells as compared to unstretched controls $(p<0.05)$; stretched cells also had significantly higher peak intensities as compared to controls $(p<0.01)$. Cells in A10T6 group showed $~ 40 \%$ increase in the total actin fluorescence intensity as compared to unstretched controls $(p<0.05)$ (Fig. 5f). These results suggest that a reinforcement of the cortical actin, caused by cyclic stretch, contributes to the increase in cellular stiffness.

\subsection{Morphoelastic model for cells under cyclic stretch and comparison with experiments}

Morphoelasticity is a mechanics-based approach used in the description of growth in biological structures. In this approach, the deformation gradient, $\mathbf{F}$, is decomposed into the product of a growth tensor, $\mathbf{G}$, to account for local addition/ removal of material, and an elastic tensor, A, to characterize reorganization within the body. The elastic part is used to ensure compatibility and integrity, and is a crucial aspect in modeling biological growth. This multiplicative decomposition is based on geometric modeling of growth such that stresses, generated in the body due to addition/ removal of material, depends on A alone. Volumetric growth in the cell, associated with mass addition, is described in our study within a finite strain elasticity framework based on deformations caused by non-uniform changes in the mass at the local level at each material point of the body (Rodriguez et al. 1994). Based on a continuum mechanics framework, we modeled the cell as a fiber-reinforced, orthotropic, hyperelastic material (Merodio and Ogden 2002; Gundiah et al. 2009). The cell is comprised of two families of SF's that grow and reorient under application of uniaxial cyclic stretch. Additional details on the theoretical model are given in the Methods (Sect. 4.8) and in Supplementary Information 2-5.

We tested the model predictions for changes in SF lengths and angles under stretch and related their influence on the 
Fig. 4 a Confocal image of an individual NIH 3T3 fibroblast cell, stained with phalloidin to visualize SF. Scale bar represents $50 \mu \mathrm{m}$. An RVE (representative volume element) of $30 \mu \mathrm{m} \times 30 \mu \mathrm{m}$ is selected to quantify stress fiber lengths within this domain. b Corresponding binarized image of the RVE with detected SF (green lines; red lines show SF with maximum length within the RVE) superimposed on the image. Scale bar represents $30 \mu \mathrm{m}$. c Mapping of detected lines as points in the Hough space $(\rho, \theta)$. d Computed lengths of the detected SF's using Hough transformation. (a)

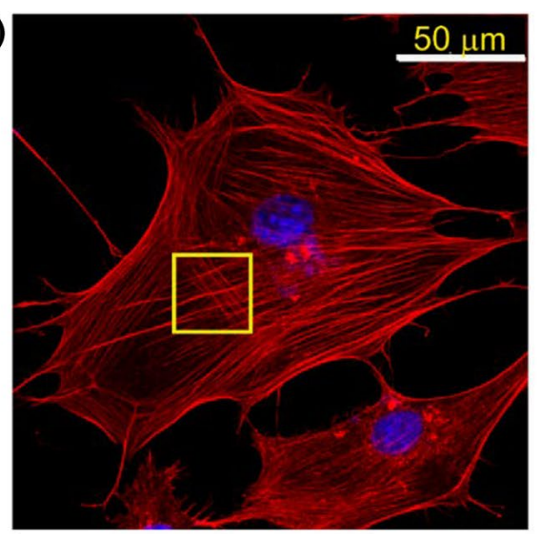

(c)

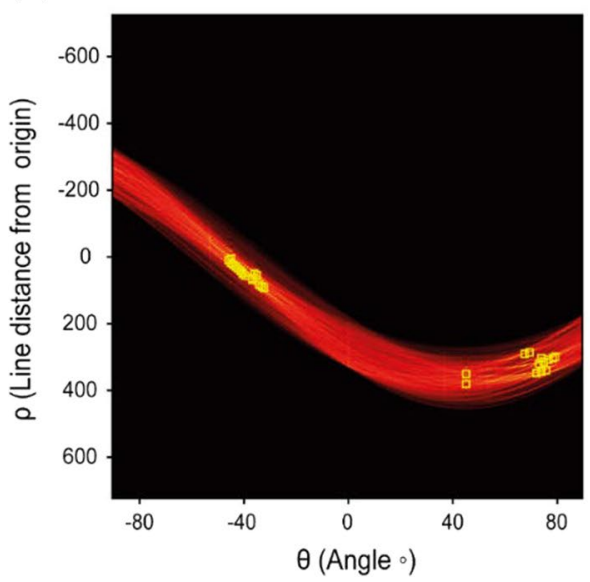

(b)

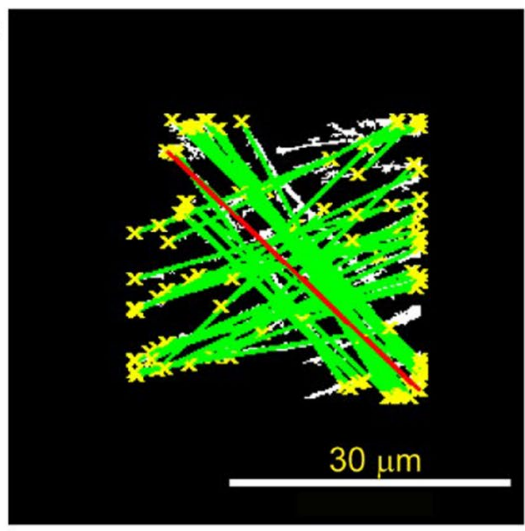

(d)

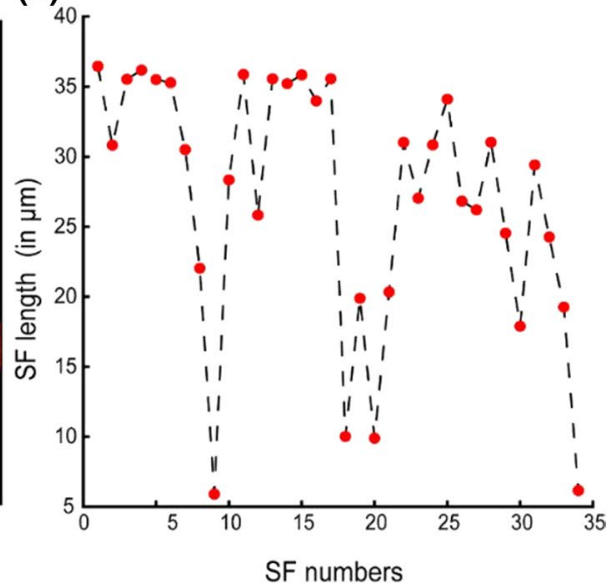

measured cell stiffness using experimental data. The evolution Eq. (3) for increase in SF length under stretch fits the experiments $\left(r^{2} \sim 0.99\right)$ (Fig. 6a). Figure 6b shows the corresponding surface plot which satisfies the thermodynamic inequality term for biological growth $\left(\boldsymbol{M}:\left(\boldsymbol{G G}^{-1}\right)>0\right)$ for $5 \%$ stretch amplitude as a function of time, and fiber angles. $\boldsymbol{M}$ is the Mandel stress tensor which is a thermodynamic conjugate to the velocity gradient, $\left(\boldsymbol{G} \boldsymbol{G}^{-1}\right)$, and is used as a natural descriptor for growth processes in open systems (Goriely 2017; Imatani and Maugin 2002). From Fig. 6b, we note that $\boldsymbol{M}:\left(\boldsymbol{G G}^{-1}\right)$ is positive to establish consistency of our growth laws for all values of time and fiber angles, and satisfies the thermodynamic constraints. The timescale for $\mathrm{SF}$ growth is described using $\tau_{\mathrm{g}}$. We explored changes in SF growth by parametrically varying $\tau_{\mathrm{g}}$ in the model (Fig. 6c, d) for the 5\% and $10 \%$ stretch amplitudes, and compared these results with experimental data. Model predictions show a good match with experimental data for $\tau_{\mathrm{g}}=2 \mathrm{~h}$.

We compared model predictions with the experimentally obtained SF orientation distributions for both 5\% and $10 \%$ stretch using the reorientation law described in Eq. 5. The theoretical model shows good agreement with experiments for increased SF orientational dynamics at higher stretch (Fig. 7a, b). Remodeling timescale for SF orientation, $\tau_{\mathrm{r}}$, changed significantly with the magnitude of applied stretch. Model predictions show a good match corresponding to $\tau_{\mathrm{r}}=3 \mathrm{~h}$ for $10 \%$ stretch and $\tau_{\mathrm{r}}=9 \mathrm{~h}$ for $5 \%$ stretch $\left(r^{2}>0.9\right.$ for both). Supplementary Fig. S3 shows parametric variations, corresponding to various initial conditions and times, for the SF reorientation law. These results clearly demonstrate that higher stretch amplitude reduced the reorientation time under cyclic stretch; this has been shown experimentally by other studies (Cui et al. 2015; Jungbauer et al. 2008) but have not been related to changes in SF lengths and changes in cellular stiffness.

We calculated changes in the effective elastic modulus, $E_{\text {eff }}$, of cells as function of fiber reorientation angle and time (Eq. 6). To account for the anisotropic fiber reinforced properties of the cell, we define the modulus, $E_{\text {eff }}$ in a given direction as the gradient of the reaction stress, $\mathbf{N}$, with respect to the stretch $(\lambda)$ in that direction evaluated for the stress-free state corresponding to $\lambda=1$, given by, $E_{\text {eff }}=\frac{\partial N(\lambda)}{\partial \lambda} \mid(\lambda=1)$ (Goriely 2017) (Supplementary Information-6). Variations in $E_{\text {eff }}$ with fiber angles $\left(\theta_{1} \in\left(0^{\circ}, 90^{\circ}\right)\right.$ and $\left.\theta_{2} \in\left(90^{\circ}, 180^{\circ}\right)\right)$ from the two SF families are shown in Fig. 8a, b. These plots show an increase in $E_{\text {eff }}$ when both fiber families align perpendicular to the applied stretch direction. Variations in $E_{\text {eff }}$ with stretching duration were also calculated for both 5\% 
and $10 \%$ stretch amplitudes using the dynamics of change in fiber orientations with time as described in Eq. 6 (Fig. 8c). These results demonstrate that the effective elastic modulus of the cell increases with SF realignment at orientations orthogonal to the direction of stretch. The model also shows that the cell modulus significantly increases after $10 \%$ cyclic stretch which correlated with the SF reorientations under stretch. Changes in cell modulus are not very significant at lower stretch amplitudes (5\% stretch) since the SF's are more randomly oriented, and not very uniformly aligned in a direction perpendicular to the applied stretch. These model predictions are in good agreement with our experimental observations of changes in cell modulus under uniaxial cyclic measured using AFM (Fig. 2f).

Finally, we calculated the variation in stresses along the direction of uniaxial cyclic stretch $\left(T_{11}\right)$ as a function the fiber orientation angles corresponding to the two SF familits $\left(\theta_{1}\right.$ and $\left.\theta_{2}\right)$ for $5 \%$ and $10 \%$ stretch amplitudes represented by the two surface plots (Fig. 8d). We observe a reduction in the intracellular stress $\left(T_{11}\right)$ with higher realignment of the SF's along a direction perpendicular to the applied stretch. We have used the overall SF orientation distributions, obtained for cells subjected to different amplitudes and durations of cyclic stretch (Table 1), to construct the structure tensors and calculate the change in stresses due to change in fiber orientation at various stretch amplitudes and time (Supplementary Information-7). Figure 8d shows the stresses $\left(T_{11}\right)$ plotted using different markers at various stretch amplitudes and time. Experimental data show a similar trend with the model, and lie very close to the surface plots. These observations are in agreement with previous models that show cyclic stretch-induced cell reorientation along the direction of minimal stress (De et al. 2007).

\section{Discussion}

Growth and dynamics of SF networks are important in maintaining cell shape, control of cellular properties, and mechanotransduction processes underlying the adhesion and migration of cells. The primary aim of this study was to quantify the morphomechanical response of fibroblasts under uniaxial cyclic stretch. There are three main implications of this work. First, we show that cyclic stretch-induced SF growth and reorientation is primarily responsible for the changes to the cellular morphometrics. SF's elongate based on the amplitude of cyclic stretch; their orientations changed from an initial random configuration in unstretched cells to a uniform direction perpendicular to the applied stretch. Cortical actin thickness also increased with applied stretch. Second, we quantified the differential roles of actin and microtubules in cellular and nuclear reorientations under stretch using submaximal concentrations of cytoskeletal inhibitors.
We show that SF reorganization is essential to the cellular orientation changes under cyclic stretch. Further, SF orientational response directly contributes to changes in the effective elastic cell modulus measured using AFM. In contrast to actin, the role of microtubules in the cytoskeleton under cyclic stretch primarily influences nuclear reorientations. Third, we use a nonlinear, orthotropic, hyperelastic, and fiber-reinforced constitutive model for the cell to elucidate the combined effects of amplitude and duration of uniaxial cyclic stretch using a growth and remodeling framework. The model uses SF evolution equations that depend on the stretch amplitude and time, and were determined experimentally to predict the effects of uniaxial cyclic stretch on SF dynamics.

Actin growth dynamics is exquisitely sensitive to mechanical stimuli; dense branched actin networks grown on functionalized surfaces demonstrate nonlinear mechanical responses (Bauer et al. 2017). Parekh et al (2005) measured the force-velocity relationships of growing actin networks in-vitro using a modified atomic force microscope (AFM) and showed the dependence of actin network growth velocity on the history of applied loading. These studies were, however, reported on in vitro preparations of isolated and purified actin networks. Our results show similar responses for actin networks in NIH $3 \mathrm{~T} 3$ fibroblast cells under stretch. We observe that the SF lengthening in fibroblasts under stretch is mechano-sensitive, depends on the stretch amplitude and time duration, and eventually reaches a steady state. We hypothesized that the mechanism of SF elongation under uniaxial cyclic stretch is mediated by stretch-activated actin polymerization (Pender and McCulloch 1991). F-actin fluorescence intensity, associated with actin polymerization, has previously been shown to increase with stretch amplitude and duration in gingival fibroblast cells (Pender and McCulloch 1991). Greiner et al. (2013) used live cell imaging techniques to demonstrate the effect of that application of uniaxial cyclic stretch on actin polymerization in fibroblasts, which leads to formation of increased cell protrusions perpendicular to the direction of stretch which initiates cell elongation orthogonal to the stretch axis.

Changes to the cellular mechanical properties associated with SF growth and remodeling, through a process of morphoelasticity, have not been demonstrated earlier. The actin cortex also plays an important role in maintaining cellular shape and maintaining deformations (Salbreaux et al. 2012; Haase and Pelling 2013). We show that the application of uniaxial cyclic stretch increases cortical actin thickness, to reinforce the cytoskeleton, that also results in an increased cell stiffness (Figs. 2f and 5d). Comparison of the cell modulus to the actin network orientations based on experimental data and model predictions demonstrates that cells with higher SF dispersions have a more compliant response, as 
(a)

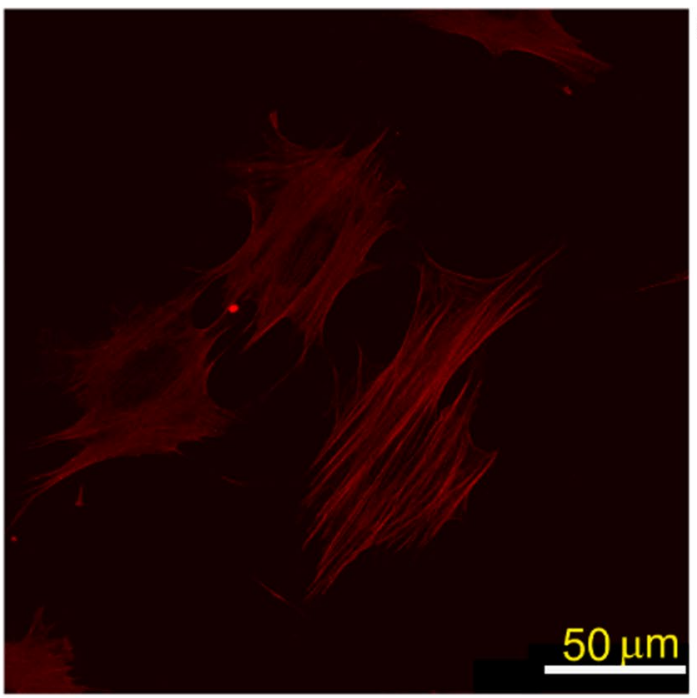

(b)

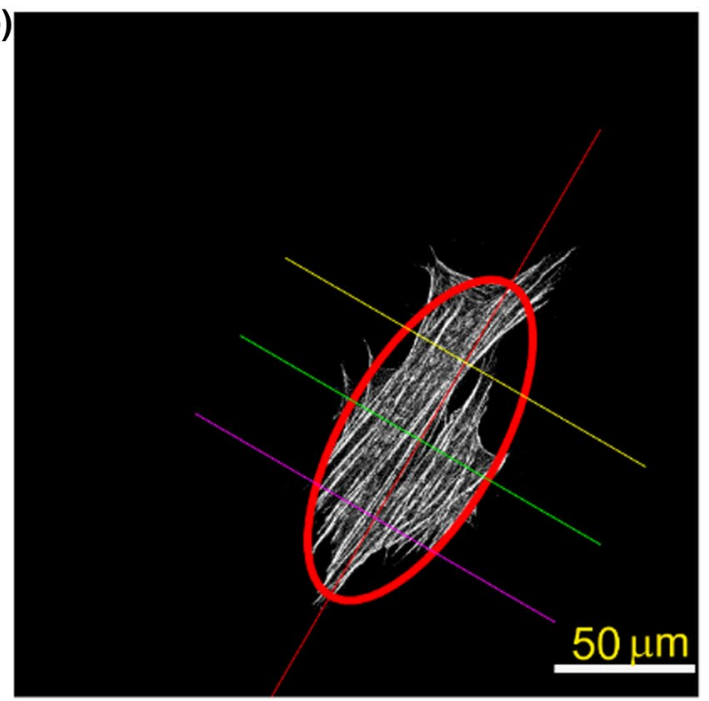

(c)
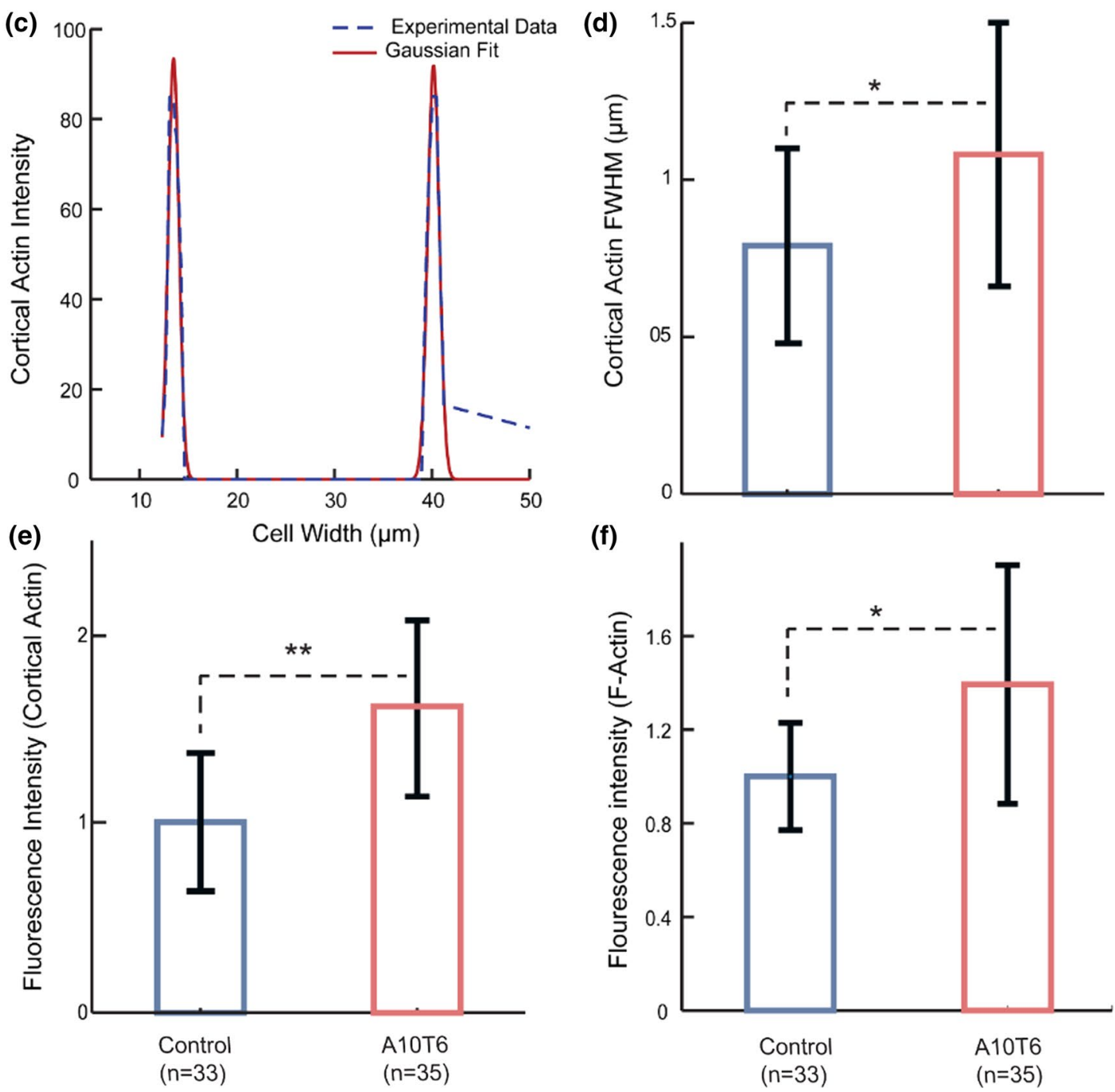
4Fig. 5 a Confocal image of NIH 3T3 fibroblast stained for F-actin (red). b Intensity distributions calculated from the binarized image at three equidistant transverse sections along the major cell axis. c Representative image of cortical actin intensity shown at one transverse section. d The full width at half maxima (FWHM) was calculated to compute the actin cortex thickness. e Peak intensity of cortical actin increased in cells subjected to cyclic stretch (A10T6). $\mathbf{f}$ The total actin fluorescence intensity in stretched cells (A10T6, $n=35$ ) increased as compared to unstretched controls $(n=33)$. Significant differences are indicated for $p<0.01(* *)$ and $p<0.05(*)$

compared to cells with highly aligned SF's (Figs. $2 \mathrm{f}$ and 8a, b) (Kulkarni et al 2018; Gavara and Chadwick 2016).

Uniaxial cyclic stretch experiments in our study highlight that the growth and realignment of SF's from a random configuration to a uniform orthogonal direction depends on the amplitude of applied external stretch which changes the overall cellular mechanical properties (Supplementary Fig. S1; Table 1). We used a phenomenological nonlinear, stretch-dependent evolution equation for SF elongation that was dependent on time and cyclic stretch amplitude (Fig. 6). We incorporated two different timescales to represent changes in the SF length and orientation under cyclic stretch. The timescale for fiber growth, $\tau_{\mathrm{g}}$, is constant and depends on the intrinsic properties of SF. $\tau_{\mathrm{r}}$ represents SF remodeling timescale which is sensitive to the applied stretch and decreases with increase in stretch amplitude (Fig. 7). We quantified the individual contributions of SF and microtubules to the cellular and nuclear reorientations under stretch, and showed that microtubule depolymerization did not significantly affect cellular reorientation. These results are in close agreement with a previous study by Goldyn et al. (2009) who showed that the dependence of force-induced cell reorientation was independent of the contributions from microtubule networks. Microtubule stability plays an important role in the kinetics of stretchinduced cellular reorientation (Goldyn et al. 2010). Coupling between microtubules and actin is critical to the cellular remodeling under stretch in human airway smooth muscle cells (Morioka et al. 2011). More recent studies show that microtubule disassembly facilitates transverse reorientation in epithelial cells, whereas inhibition of myosin II results in cell orientation along the direction of stretch (Lien and Wang 2021). AFM indentation experiments in our study also show that the depolymerization of microtubules did not change the increase in cellular stiffness under stretch. Microtubule disruption with nocodazole results in increased RhoA activation and a corresponding increase in SF formation and anisotropy perpendicular to the direction of cyclic stretch (Goldyn et al. 2010; Foolen et al. 2014). Studies also show that nocodazole treatment increases myosin phosphorylation and enhances cellular contractility (Kolodney and Elson 1995; Morioka et al 2011). The increased stiffness of nocodazole treated cells under stretch in our study may hence be related to these molecular mechanisms. Formation and re-organization of SF's under stretch were, however, critical in the stretch-induced cytoskeletal stiffening. Recent studies show that reduction in actin-myosin II interactions in smooth muscle cells (SMCs) enhances cyclic stretchinduced SF disassembly (Huang et al. 2021). Intermediate filaments also play an important role in the dynamics of cell reorientation and the cellular mechanical properties under the application of uniaxial cyclic stretch (Wang and Stamenovic 2000; Zielinski et al. 2018; Leccia et al. 2013). Vimentin deficient fibroblasts exhibit a softer mechanical response as compared to control cells (Wang and Stamenovic 2000). Vimentin networks in endothelial cells also reorient along a direction perpendicular to the applied stretch (Zielinski et al. 2018). Desmin is critical in determining the morphology, cell spread area, and the reorientation of myoblasts under cyclic stretch (Leccia et al. 2013).

Stress or strain-based homeostasis in cells is hypothesized to be important in cyclic stretch-induced cytoskeletal reorganization (De et al. 2007; Kaunas et al. 2010). Chen et al. modeled changes in SF orientations based on the varying oscillatory stretches at focal adhesions in the cell (Chen et al. 2012). Cell reorientation under cyclic stretch has been studied in recent years; however, the context of evolution of cellular morphometrics due to this active reorientation process remains underexplored. SFs are generally classified into four categories as ventral and dorsal SF, transverse arcs, and perinuclear actin cap (Livne and Geiger 2016). We have not considered the effects of branched networks in this study, and have only considered the ventral SF in this study. FAs destabilize under oscillatory forces and slide or relocate on the membrane that may result in SF reorientations and length changes (Greiner et al. 2013). We have also not considered the dynamic effects of focal adhesion remodeling which is also a limitation in our study.

In conclusion, we used a novel growth and remodeling framework to characterize the effects of uniaxial cyclic stretch in fibroblasts using experimental stretching methods in combination with a continuum mechanics framework. We show that an increase in the cellular stiffness under cyclic stretch is linked to SF growth and reorientation in a direction orthogonal to the direction of stretch. The theoretical model provides new understanding on a well-observed phenomenon of cell re-orientation under cyclic stretch that we show is linked to the growth of SF and their orientation. Such studies demonstrate the importance of uniaxial stretching in mechanotransduction processes, related to changes in the cytoskeleton, which are essential in disease models for aneurysm growth and fibrosis that use a qualitative representation of the cell under stretch. 
Fig. 6 a Changes in SF lengths with time (h) under 5\% and $10 \%$ amplitude cyclic stretch calculated from experiments (Mean \pm SEM) used to obtain the SF growth evolution law ( $n \sim 30$ in each group). b Thermodynamic inequality for growth was assessed as a function of stretch duration and SF orientation. $\mathbf{c}$ and $\mathbf{d}$ The growth timescale $\left(\tau_{\mathrm{g}}\right)$ was parametrically varied for $5 \%$ (black) and $10 \%$ stretch (red)
Fig. 7 a and b Comparisons of the computed SF reorientation under cyclic stretch with experiments show the relation between SF remodeling timescale $\left(\tau_{\mathrm{r}}\right)$ and stretch amplitude. $\tau_{\mathrm{r}}$ was $9 \mathrm{~h}$ for $5 \%$ and $3 \mathrm{~h}$ for $10 \%$ uniaxial cyclic stretch, respectively (a)

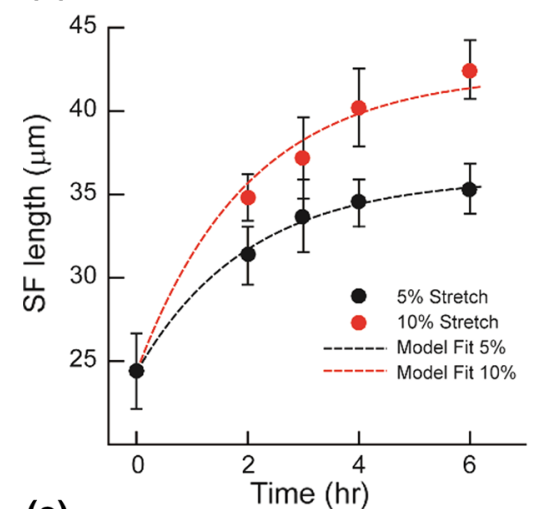

(c)

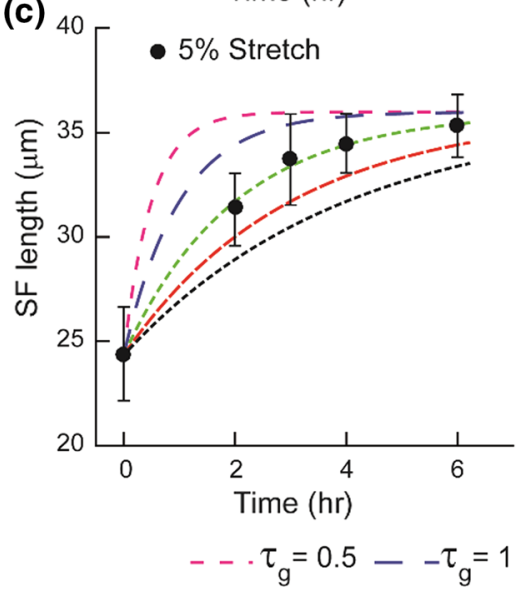

(b)

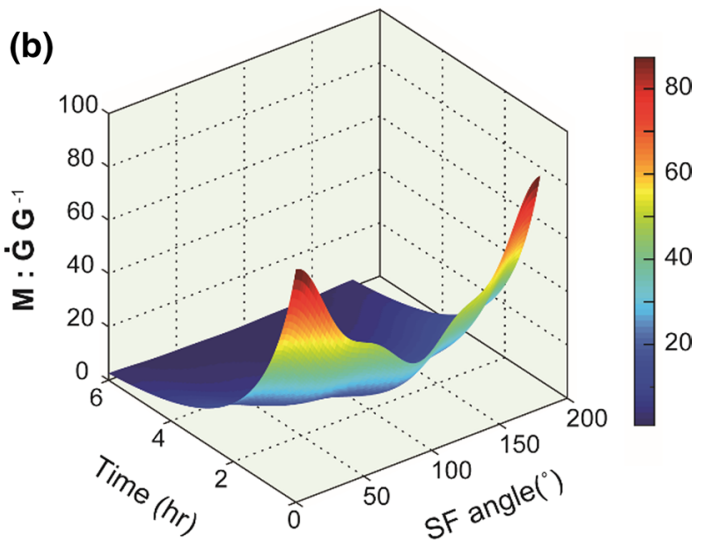

(d)

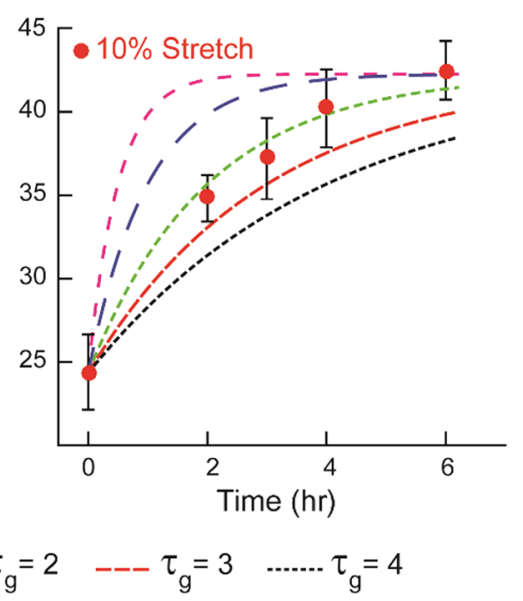

(a)

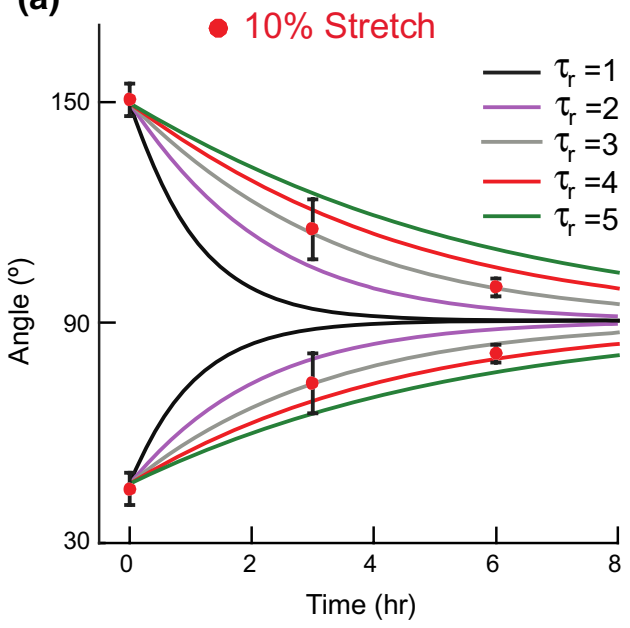

(b)

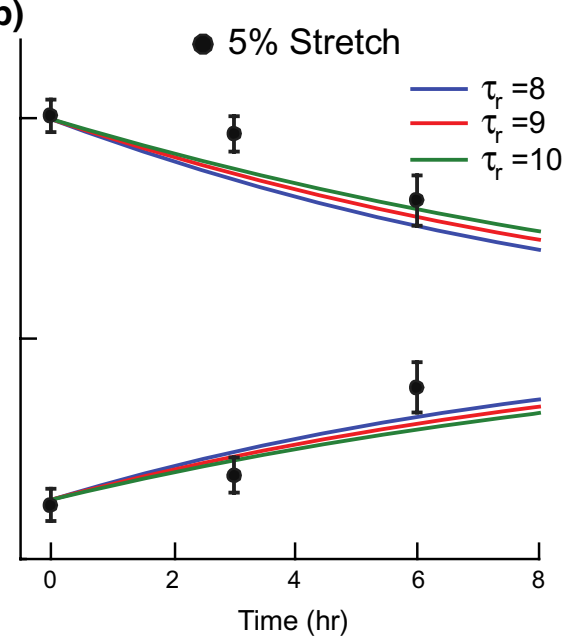

\section{Materials and methods}

\subsection{Design and assembly of the bioreactor for cell stretching}

The custom designed cell stretcher allows for both uniaxial and biaxial stretching with equal and different strain rates on each actuator. We designed a novel clamping arrangement to clamp a thin stretchable membrane (width $10 \mathrm{~mm} \times$ length $20 \mathrm{~mm} \times$ thickness $150 \mu \mathrm{m}$ ) between two opposite translating actuator arms located within a custom bioreactor for the dynamic stretch experiments. The clamps were $3 \mathrm{D}$ printed using a biocompatible 
Fig. 8 a and b Variations in $E_{\text {eff }}$ for the cell, subjected to $5 \%$ and $10 \%$ amplitude cyclic stretch, as a function of SF alignment angle. $\mathbf{c}$ Changes in $E_{\text {eff }}$ from the model as a function of SF reorientation under cyclic stretch are shown for $5 \%$ (red) and $10 \%$ (blue) stretch. d Changes in cell tractions $\left(T_{11}\right)$ under stretch from the model were compared with experimental results
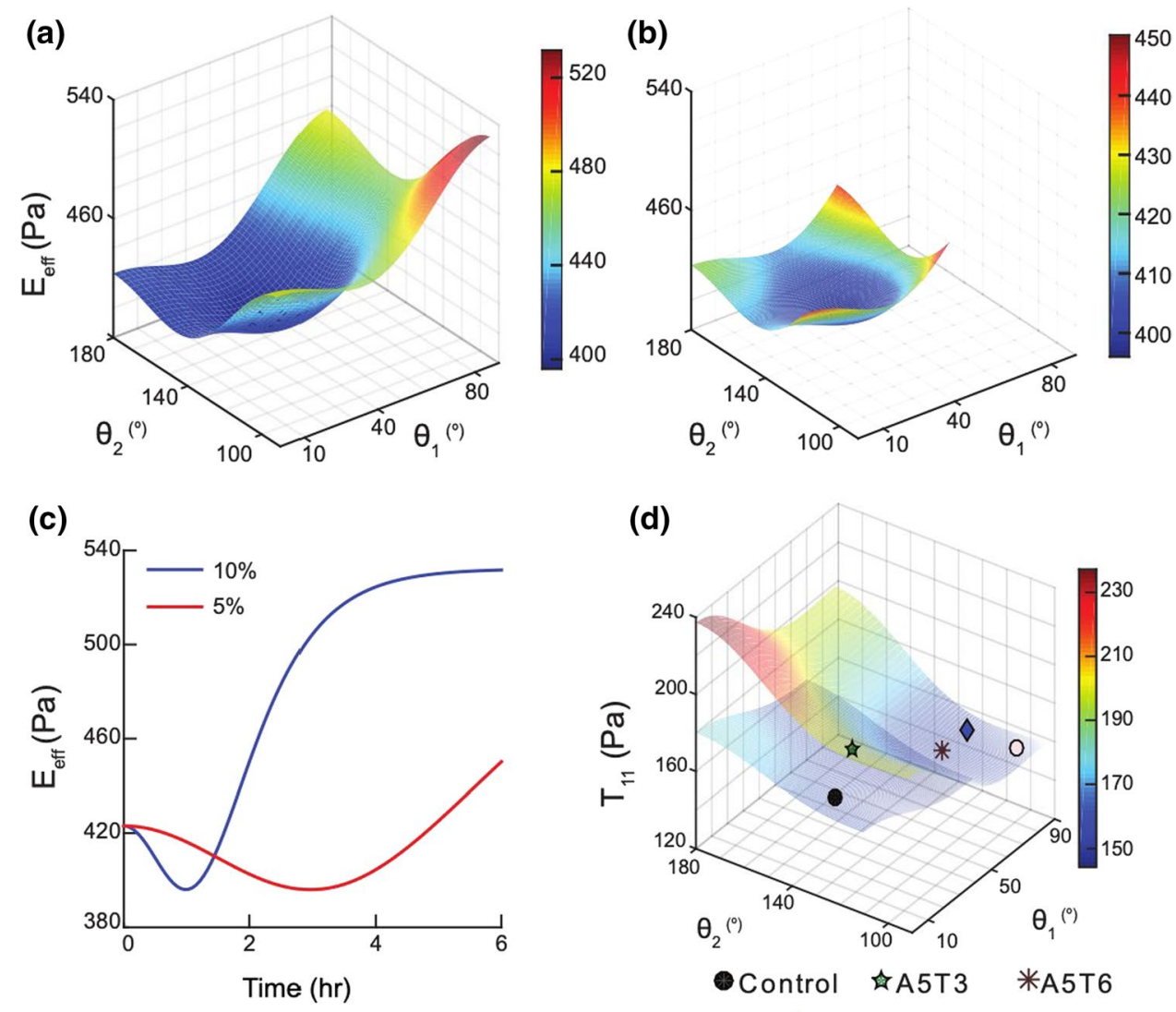

acrylic compound (Verro White; Stratasys) (Fig. 1a, b). The actuator arms were connected to a controller for the motorized biaxial stretching device (BiSS, India) and operated using user-controlled stretch amplitude and frequency inputs over extended time periods. The entire setup was placed within a humidified temperaturecontrolled incubator at $37{ }^{\circ} \mathrm{C}$ and $5 \% \mathrm{CO}_{2}$ to maintain sterility.

\subsection{Strain quantification}

In-plane strains were quantified by tracking fiduciary markers in the PDMS (poly di-methyl siloxane; Sylgard (1184, Dow Corning) sheet, mixed in the ratio of 10:1 elastomer to curing agent, using an overhead video camera (Sony HD HDR-XR100E). Marker displacements from the referential to the deformed configuration were measured and the deformation gradient, $\mathbf{F}$, was computed as $\boldsymbol{F}=\boldsymbol{I}+\boldsymbol{H}$, where $\boldsymbol{H}=\partial \boldsymbol{u} / \partial \boldsymbol{X}$ is the displacement gradient in the referential configuration. A strain interpolation algorithm, implemented using MATLAB (v R2016a), was used to quantify in-plane Green-Lagrange strains in the specimen using measured values of the deformation gradients (Fig. 1c) (Gundiah et al. 2009; Agrawal et al. 2013).

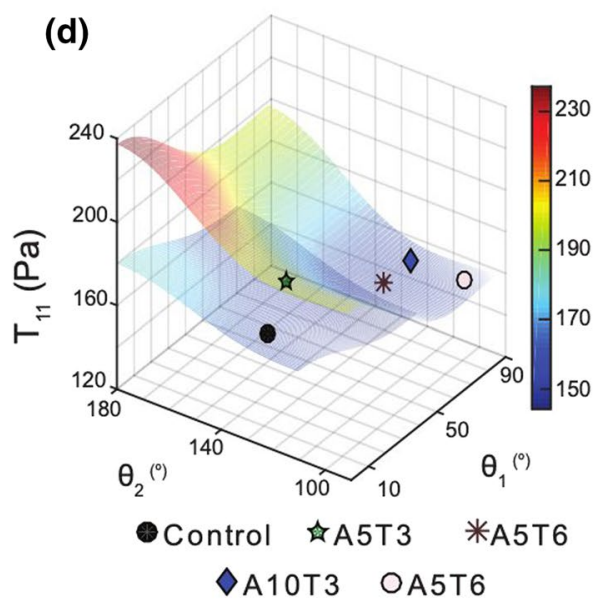

\subsection{Cell culture and immunofluorescence staining}

NIH 3 T3 fibroblast cells, obtained from ATCC (ATCC® CRL-1658 ${ }^{\mathrm{TM}}$ ) and cultured in Dulbecco's minimum essential medium (DMEM; Sigma-Aldrich) supplemented with $10 \%$ FBS (GIBCO BRL) and 1 vol\% of penicillin-streptomycin (Sigma-Aldrich), were maintained in a humidified temperature-controlled incubator (Panasonic Inc.) at $37{ }^{\circ} \mathrm{C}$ and $5 \% \mathrm{CO}_{2}$. Following stretching experiments, cells were fixed in $4 \%$ paraformaldehyde, permeabilized in $0.5 \%$ TritonX-100 (Sigma) solution for $10 \mathrm{~min}$, and stained either for actin using rhodamine phalloidin (Thermofisher 1:200) or microtubules using $\alpha$-tubulin FITC (Invitrogen 1:200), and a nuclear DAPI counterstain (Thermofisher $1: 400)$. Samples were imaged using a confocal microscope (Leica Microsystems, TCS SP5 II) using 10X, and $63 \mathrm{X}$ oil immersion objectives to visualize the cytoskeletal organization. 


\subsection{Uniaxial cyclic stretching of fibroblasts in the bioreactor and cytoskeletal inhibitor treatments}

Thin flexible PDMS membranes, mixed in the ratio of 10:1 elastomer to curing agent, coated with fibronectin were used as substrates to culture cells. Prepared substrates were incubated with DMEM containing 10\% FBS for $30 \mathrm{~min}$, and followed by seeding of cells. Cell-seeded constructs were incubated overnight at $37{ }^{\circ} \mathrm{C}$ and $5 \% \mathrm{CO}_{2}$ to allow cell attachment, clamped to two translating actuator arms as described earlier, and were stretched cyclically (5\% and $10 \%$ amplitude) at $1 \mathrm{~Hz}$ for varied durations to explore the temporal dependence of stretching on cell alignment $(3 \mathrm{~h}$ and $6 \mathrm{~h}$ ). The bioreactor was filled with cell culture medium to ensure cell viability through the duration of the experiment.

To delineate the individual contributions of actin and microtubule in cellular response under cyclic stretch, we treated cells with either $0.5 \mu \mathrm{M}$ concentration of cytochalasin-D (Sigma-Aldrich; USA) to depolymerize actin, or $0.5 \mu \mathrm{M}$ concentration nocodazole (Sigma-Aldrich) to disrupt microtubules (Barreto et al. 2013). Submaximal concentrations of the cytoskeletal disruptors were used in our experiments to prevent cellular detachment from the basement membrane. Fibroblasts have long and well-defined SF's in contrast to short, choppy actin fibers following cytochalasin$\mathrm{D}$ treatment, which result in a distinct change in cellular morphology. We ensured that the same concentration of the drug was present during the stretching and AFM indentation experiments to prevent possible repolymerizations of actin and microtubules that may contribute to possible artifacts in the experiments.

\subsection{Quantification of SF orientation under cyclic stretch}

Confocal images of cells stained for actin were analyzed using a custom MATLAB program (Mathworks, 2016b) (Schriefl et al. 2013; Kulkarni et al 2018) (Fig. 3a). The binarized image of actin in the cell was obtained and represented using a distribution function $f(x, y) ;(x, y)$ defines a point in the 2-D image (Fig. 3b). We calculated the fast Fourier transformation (FFT) of this image as $\mathscr{F}(f(x, y))=(u, v)$ where $(u, v)$ denotes a point in the Fourier space. The power spectrum of this matrix was obtained by $P(u, v)=\mathscr{F}(u, v)$. $\mathscr{F} *(u, v), \mathscr{F} *$ is the complex conjugate of the function, $F$ in this expression (Fig. 3c). The SFs were distinguished based on the spatial frequencies and orientations in Fourier space. A wedge-shaped orientation filter was used to quantify the SF orientation distributions (Kulkarni et al. 2018). The histogram for angular orientation of SF for each cell was fit to a two-peak Gaussian distribution to quantify the mean SF orientations (Fig. 3d). We obtained histograms of
SF orientations for $\sim 30$ cells in each group, and used them to calculate the global peak-average angular distributions for cells subjected to different amplitudes and durations of cyclic stretch.

\subsection{Quantification of SF lengths}

We used Hough transforms in a custom MATLAB program (Mathworks, 2016b) to compute the SF lengths using confocal images of cells stained for actin (Hough 1962). SF edges were detected using a binarized image of actin fibers in the cell with the Canny edge detector (Fig. 4a, b). The edge points were next mapped to Hough space and stored in an accumulator (Fig. 4c). Data from all coordinates corresponding to all points in the accumulator were used to compute the fiber lengths (Fig. 4d).

The efficiency of fiber detection in confocal images using Hough transform is sensitive to a variety of parameters which include the magnification of the images, fluorescence intensity, and also the thresholding and segmentation of the images. Based on our confocal images, these parameters are standardized for improved SF length detection. In this method, we set the minimum length of the detected SF to be $4 \mu \mathrm{m}$, and neglected segments which were below this value (Fig. 4). To test the accuracy of this method in detecting fiber angles and lengths, we created a synthetic network of lines with pre-defined lengths at various orientation. Comparisons between the results from the Hough transform method implemented in this work were in good agreement with the actual measurements (Supplementary Fig. S2). A limitation of this method is in the quantification of curved lines that are not relevant in the context of SF.

\subsection{Measurement of effective modulus of the cell using atomic force microscope (AFM)}

We used a Park Systems XE-Bio AFM to quantify the effective modulus of the cell using an App Nano Hydra 6V-200NGTL silicon dioxide cantilever (stiffness $\sim 0.045 \mathrm{~N} / \mathrm{m}$ ) with an attached spherical bead of diameter $5.2 \mu \mathrm{m}$. The stiffness of the cantilever was determined to be $0.041 \mathrm{~N} / \mathrm{m}$ using thermal tuning method. Specific locations for indentations in the cell were selected based on the cell topography, and were equidistant from the nucleus and the cell edge to minimize the influence of the nucleus. Indentation experiments were performed using cells in Hank's balanced salt solution, and the corresponding force-displacement curves for each location were used to quantify the effective modulus of the cell using the material properties of the cantilever and a thin layer, finite thickness Hertzian contact mechanics model (Darling et al 2007; Dimitradis et al. 2002). The force curve, corresponding to the approach of the tip toward the substrate, was measured and a constant indentation depth of $1 \mu \mathrm{m}(\sim 14 \%$ of the total 
cell height) was maintained for all data, which lies within the 4-30\% range of the cell height reported in the literature, that are required for the equations to be used for such studies.

\subsection{Morphoelastic model of the cell under stretch}

Let $\mathbf{F}$ be the deformation gradient used to describe the cell undergoing stretch from an unstretched reference configuration. We model the growth of SF's in the fibroblast under stretch using the growth tensor, $\mathbf{G}$, which is a virtual stress-free configuration. The kinematics of SF growth is accompanied with an elastic deformation, A. We write,

$\mathbf{F}=\mathbf{A} \cdot \mathbf{G}$

Coupling between the elastic responses and biological growth is integral in morphoelasticity and is consistent from kinematic and thermodynamic perspectives. Results from experiments to quantify the length changes of SF in fibroblasts under uniaxial cyclic stretch were used to determine the growth tensor $\mathbf{G}$ based on the evolving configurations during cell reorientation under stretch. The elastic deformation tensor, A, depends on the externally applied cyclic stretch amplitude that is measured using the strain quantification experiments (Fig. 1c).

We model the cell as a fiber-reinforced orthotropic hyperelastic material, comprised of two families of SF, based on the microscopy images (Supplementary Fig S1, Fig. 3) (Merodio and Ogden 2002; Gundiah et al 2009). SF angular orientations result in anisotropic cellular mechanical responses that are described using a continuum mechanical description for the cell using terms to account for fiber orientations (Efremov et al. 2019, Sakamoto et al., 2017). A representative element of the cell is characterized through dependence on $\mathbf{A}$ and is given by the strain energy function, $W(\boldsymbol{A})$ (Supplementary Information-2).

$W(A)=W_{\text {iso }}+W_{\text {aniso }}=\frac{\mu}{2}\left(I_{1}-3\right)+\frac{\mu \alpha}{2}\left[\left(I_{4}-1\right)^{2}+\left(I_{6}-1\right)^{2}\right]$

$\mu$ is the shear modulus $(\mu>0)$ and $\alpha$ is a measurement of the additional fiber reinforcement $(\alpha>0)$.

We quantified SF lengths for individual cells in each data set corresponding to different stretch amplitudes (at 5\% and $10 \%)$ and duration $(2,3,4$, and $6 \mathrm{~h}$ ) and averaged them to compute an overall SF length distribution (Supplementary Table 1). We used these experimental data to formulate a new evolution equation for SF length increase $(\gamma)$ as a function of the applied stretch, $\lambda$, and time, $t$. The evolution equation is given by

$\gamma(\lambda, t)=f(\lambda) g(t)+c$

where $f(\lambda)=25.23\left(1-e^{-12.39(\lambda-1)}\right) \quad$ and $\quad g(t)=\left(1-e^{-t / \tau_{g}}\right)$

$c$ is a constant and has a value $24.4 \pm 2.3 \mu \mathrm{m}$ (Mean \pm SEM) in unstretched (control) cells; $\tau_{\mathrm{g}}$ is a timescale for the growth of SF. $f(\lambda)$ is constant for a given stretch amplitude and is independent of time.

This form of the growth law is similar to a special case of the four-parameter Richards growth model (Tjorve and Tjorve 2010) given by

$W(t)=a\left(1-\left(\frac{1}{d}\right) \cdot\left(e^{-K_{\mathrm{g}}\left(t-T_{i}\right)}\right)^{d}+W_{0}\right.$

where $K_{\mathrm{g}}$ is the model-specific growth constant that controls the maximum growth rate. This term is similar to $1 / \tau_{\mathrm{g}}$ in our model. The term, a, represents the upper asymptote and is equivalent to the value of $f(\lambda) . W_{0}$ represents the initial value and is similar to $\mathrm{c}$ in our model. $T_{i}$, with units of time, is referred to as a location parameter that shifts the growth curve horizontally and is excluded in our model for simplicity. The parameter, $d$, controls the inflection value of the curve. We parametrically varied the parameter $d$ in the Richards model (Eq. 4) for 5\% and 10\% stretch amplitude and compared these results with experimental data. Supplementary Fig. 4 shows equivalence between the model corresponding to $d=1$ to the SF growth law (Eq. 3) in our study.

Coefficients in $f(\lambda)$ were obtained by fitting the experimental data to Eq. (3). The evolution equation for SF lengthening shows that $\gamma$ increases with increase in both applied stretch, $\lambda$, and time duration, $t$, and eventually reaches equilibrium. We use these evolution equations to construct the growth tensor $\mathbf{G}$ in the context of pure fiber growth for a single SF as

$\boldsymbol{G}_{r-\theta-z}=\boldsymbol{I}+(\gamma-1) \boldsymbol{e}_{r} \otimes \boldsymbol{e}_{r}$

$\boldsymbol{e}_{r}$ is the unit vector in SF growth direction. The form of the growth tensor chosen in this study is symmetric and has been used in previous studies (Goktepe et al. 2010). This reduces the number of growth descriptors for anisotropic growth in analytic treatments. We next transform the growth tensor from the cylindrical $(r-\theta-z)$ coordinate system to Cartesian (Eq. 17 in Supplementary Information 3). We tested the consistency of our growth law by including thermodynamic restrictions for active biological growth subjected to various possible fiber angles and experimental duration for different stretch amplitudes based on the Clausius-Duhem inequality using the standard Coleman-Noll procedure (Supplementary Information 3,4).

We assume a form for the SF re-orientation equations along a uniform direction perpendicular to applied cyclic uniaxial stretch, corresponding to $X$-axis, as 


$$
\frac{d\left(\boldsymbol{m}_{\boldsymbol{o}}^{i}\right)}{d t}=\frac{1}{\tau_{r}}\left(\boldsymbol{n}-\left(\boldsymbol{n} \cdot \boldsymbol{m}_{\boldsymbol{o}}^{i}\right) \boldsymbol{m}_{\boldsymbol{o}}^{i}\right)
$$

The vector $\mathbf{n}$ represents the unit vector along the direction of preferred cytoskeletal orientation orthogonal to the applied stretch in the $Y$-axis $\left(\mathbf{e}_{2}\right)$ (Melnik and Goriely 2013; Menzel 2005). $\tau_{r}$ is the remodeling timescale which depends on the stretch amplitude (Supplementary Information-5). The elongation and reorientation dynamics of SF in cells for $10 \%$ stretch amplitude over $6 \mathrm{~h}$ (A10T6) is shown in Supplementary Video 1.

Supplementary Information The online version contains supplementary material available at https://doi.org/10.1007/s10237-021-01548-z.

Acknowledgements We thank Ms. Monisha Mohandas (BSSE, IISc) for help with the AFM. We also thank Ms. Dhulika Ravinuthala who helped in performing some of the uniaxial cyclic stretch experiments reported in this study. NG gratefully acknowledges the Department of Biotechnology (BBI2) and Department of Science and Technology (SERB/003640) for project support. PK laboratory is supported by IISc-DBT partnership program and DST-FIST infrastructure to MRDG.

Author contributions AC performed the experiments, analyzed the data, completed the theoretical model, and helped write the manuscript. PK provided inputs on the data interpretation and results. NG designed the study, supervised the research, helped with data analysis, and wrote the manuscript with inputs from all authors.

\section{References}

Agrawal V, Kollimada SA, Byju AG, Gundiah N (2013) Regional variations in the nonlinearity and anisotropy of bovine aortic elastin. Biomech Model Mechanobiol 12:1181-1194

Balaban NQ, Schwarz US, Riveline D, Goichberg P, Tzur G, Sabanay I, Mahalu D, Safran S, Bershadsky A, Addadi L, Geiger B (2001) Force and focal adhesion assembly: a close relationship studied using elastic micropatterned substrates. Nat Cell Biol 3(5):466-472

Barreto S, Clausen CH, Perrault CM, Fletcher DA, Lacroix D (2013) A multi-structural single cell model of force-induced interactions of cytoskeletal components. Biomaterials 34(26):6119-6126

Bauër P, Tavacoli J, Pujol T, Planade J, Heuvingh J, Du Roure O (2017) A new method to measure mechanics and dynamic assembly of branched actin networks. Sci Rep 1:15688

Buck RC (1980) Reorientation response of cells to repeated stretch and recoil of the substratum. Exp Cell Res 127(2):470-474

Chen B, Kemkemer R, Deibler M, Spatz J, Gao H (2012) Cyclic stretch induces cell reorientation on substrates by destabilizing catch bonds in focal adhesions. PLoS ONE 7(11):48346

Chen Y, Pasapera AM, Koretsky AP, Waterman CM (2013) Orientation-specific responses to sustained uniaxial stretching in focal adhesion growth and turnover. Proc Natl Acad Sci USA 110(26):E2352-E2361

Chen K, Vigliotti A, Bacca M, McMeeking RM, Deshpande VS, Holmes JW (2018) Role of boundary conditions in determining cell alignment in response to stretch. Proc Natl Acad Sci USA 115(5):986-991

Cirka H, Monterosso M, Diamantides N, Favreau J, Wen Q, Billiar K (2016) Active traction force response to long-term cyclic stretch is dependent on cell pre-stress. Biophys J 110(8):1845-1857

Clark AG, Dierkes K, Paluch EK (2013) Monitoring actin cortex thickness in live cells. Biophys J 105:570-580. https://doi.org/ 10.1016/j.bpj.2013.05.057

Cui Y, Hameed MF, Yang B, Lee K, Pan CQ, Park S, Sheetz M (2015) Cyclic stretching of soft substrates induces spreading and growth. Nat Commun 6(1):6333

Darling EM, Zauscher S, Block JA, Guilak FA (2007) Thin-layer model for viscoelastic, stress-relaxation testing of cells using atomic force microscopy: Do cell properties reflect metastatic potential? Biophys J 92(5):1784-1791

De R, Zemel A, Safran SA (2007) Dynamics of cell orientation. Nat Phys 3(9):655-659

Dimitriadis EK, Horkay F, Maresca J, Kachar B, Chadwick RS (2002) Determination of elastic moduli of thin layers of soft material using the atomic force microscope. Biophys J 82(5):2798-2810

Efremov YM, Velay-Lizancos M, Weaver CJ, Athamneh AI, Zavattieri PD, Suter DM, Raman A (2019) Anisotropy vs isotropy in living cell indentation with AFM. Sci Rep 9:5757

Foolen J, Marloes WJT, Broek JVD, Baaijens F (2014) Synergy between Rho signaling and matrix density in cyclic stretchinduced stress fiber organization. Acta Biomater 10:1876-1885

Gavara N, Chadwick RS (2016) Relationship between cell stiffness and stress fiber amount, assessed by simultaneous atomic force microscopy and live-cell fluorescence imaging. Biomech Model Mechanobiol 15(3):511-523

Göktepe S, Abilez OJ, Parker KK, Kuhl E (2010) A multiscale model for eccentric and concentric cardiac growth through sarcomerogenesis. J Theor Biol 265(3):433-442

Goldyn AM, Rioja BA, Spatz JP, Ballestrem C, Kemkemer R (2009) Force-induced cell polarisation is linked to RhoA-driven microtubule-independent focal-adhesion sliding. J Cell Sci 122(20):3644-3651

Goldyn AM, Kaiser P, Spatz JP, Ballestrem C, Kemkemer R (2010) The kinetics of force-induced cell reorganization depend on microtubules and actin. Cytoskeleton 67:241-250

Goriely A (2017) The Mathematics and mechanics of biological growth. Springer, New York. https://doi.org/10.1007/ 978-0-387-87710-5

Greiner AM, Chen H, Spatz JP, Kemkemer R (2013) Cyclic tensile strain controls cell shape and directs actin stress fiber formation and focal adhesion alignment in spreading cells. PLoS ONE. https://doi.org/10.1371/journal.pone.0077328

Gundiah N, Ratcliffe MB, Pruitt LA (2009) The biomechanics of arterial elastin. J Mech Behav Biomed Mater 2(3):288-296

Haase K, Pelling AE (2013) The role of the actin cortex in maintaining cell shape. Commun Integr Biol 6(6):e26714

Hayakawa K, Sato N, Obinata T (2001) Dynamic reorientation of cultured cells and stress fibers under mechanical stress from periodic stretching. Exp Cell Res 268(1):104-114

Hoffman L, Jensen CC, Yoshigi M, Beckerle M (2017) Mechanical signals activate p38 MAPK pathway-dependent reinforcement of actin via mechanosensitive HspB1. Mol Biol Cell 28(20):2661-2675

Hough PVC (1962) Method and means for recognizing complex patterns. US Patent 3,069,654

Hsu HJ, Lee CF, Locke A, Vanderzyl SQ, Kaunas R (2010) Stretchinduced stress fiber remodeling and the activations of JNK and ERK depend on mechanical strain rate, but not FAK. PLoS ONE 5(8): 12470

Huang W, Matsui ST, Saito T, Kuragano M, Takahashi M, Kawahara T, Sato M, Deguchi S (2021) Mechanosensitive myosin II but not 
cofilin primarily contributes to cyclic cell stretch-induced selective disassembly of actin stress fibers. Am J Physiol Cell Physiol 320:C1153-C1163

Humphrey JD, Rajagopal KR (2002) A constrained mixture model for growth and remodeling of soft tissues. Math Model Methods Appl Sci 12(03):407-430

Imatani S, Maugin GA (2002) Constitutive model for material growth and its application to three-dimensional finite element analysis. Mech Res Commun 29(6):477-483

Jungbauer S, Gao H, Spatz JP, Kemkemer R (2008) Two characteristic regimes in frequency-dependent dynamic reorientation of fibroblasts on cyclically stretched substrates. Biophys J 95(7):3470-3478

Kaunas R, Nguyen P, Usami S, Chien S (2005) Cooperative effects of Rho and mechanical stretch on stress fiber organization. Proc Natl Acad Sci USA 102(44):15895-15900

Kaunas R, Hsu HJ, Deguchi S (2010) Sarcomeric model of stretchinduced stress fiber reorganization. Cell Health Cytoskelet 3:13

Kolodney MS, Elson EL (1995) Contraction due to microtubule disruption is associated with increased phosphorylation of myosin regulatory light chain. Proc Natl Acad Sci USA 92:10252-10256

Kulkarni AH, Chatterjee A, Kondaiah P, Gundiah N (2018) TGF- $\beta$ induces changes in breast cancer cell deformability. Phys Biol 15(6):065005

Leccia E, Batonnet-Pichon S, Tarze A, Bailleux V, Doucet J, Pelloux M, Delort F, Pizon V, Vicart P, Briki F (2013) Cyclic stretch reveals a mechanical role for intermediate filaments in a desminopathic cell model. Phys Biol 10:016001

Lien JC, Wang YL (2021) Cyclic stretching-induced epithelial cell reorientation is driven by microtubule-modulated transverse extension during the relaxation phase. Sci Rep 11:14803

Livne A, Geiger B (2016) The inner workings of stress fibers-from contractile machinery to focal adhesions and back. J Cell Sci 129(7):1293-1304

Livne A, Bouchbinder E, Geiger B (2014) Cell reorientation under cyclic stretching. Nat Commun 5:1-8

Melnik AV, Goriely A (2013) Dynamic fiber reorientation in a fiber-reinforced hyperelastic material. Math Mech Solids 18(6):634-648

Menzel A (2005) Modelling of anisotropic growth in biological tissues. Biomech Model Mechanobiol 3(3):147-171

Merodio J, Ogden RW (2002) Material instabilities in fiber-reinforced nonlinearly elastic solids under plane deformation. Arch Mech 54(5-6):525-552

Morioka M, Parameswaran H, Naruse K, Kondo M, Sokabez M, Hesegawa Y, Suki B, Ito S (2011) Microtubule dynamics regulate cyclic stretch-induced cell alignment in human airway smooth muscle cells. PLoS ONE 6(10):e26384

Nekouzadeh A, Pryse KM, Elson EL, Genin GM (2008) Stretch-activated force shedding, force recovery, and cytoskeletal remodeling in contractile fibroblasts. J Biomech 41(14):2964-2971
Parekh SH, Chaudhuri O, Theriot JA, Fletcher DA (2005) Loading history determines the velocity of actin-network growth. Nat Cell Biol 7(12):1119-1123

Pender N, McCulloch CA (1991) Quantitation of actin polymerization in two human fibroblast sub-types responding to mechanical stretching. J Cell Sci 100:187-193

Perrault CM, Brugues A, Bazellieres E, Pierre Ricco P, Lacroix D, Trepat X (2015) Traction forces of endothelial cells under slow shear flow. Biophys J 109(8):1533-1536

Robertson AM, Watton PN (2013) Mechanobiology of the arterial wall. Transp Biol Media, pp 275-347

Rodriguez EK, Hoger A, McCulloch AD (1994) Stress-dependent finite growth in soft elastic tissues. J Biomech 27(4):455-467

Sakamoto Y, Buchanan RM, Sanchez-Adams J, Guilak F, Sacks MS (2017) On the functional role of valve interstitial cell stress fibers: a continuum modeling approach. J Biomech Eng 139:021007

Salbreux G, Charras G, Paluch E (2012) Actin cortex mechanics and cellular morphogenesis. Trends Cell Biol 22:536-545

Schriefl AJ, Reinisch AJ, Sankaran S, Pierce DM, Holzapfel GA (2013) Quantitative assessment of collagen fibre orientations from twodimensional images of soft biological tissues. J R Soc Interface 9(76):3081-3093

Thompson DW (1917) On growth and form. Cambridge University Press, Cambridge

Tjorve E, Tjorve MCK (2010) A unified approach to the Richardsmodel family for use in growth analyses: why we need only two model forms. J TheoR Biol 2010(267):417-425

Wang N, Stamenovic D (2000) Contribution of intermediate filaments to cell stiffness, stiffening, and growth. Am J Physiol Cell Physiol 279:188-194

Wang JHC, Goldschmidt CP, Wille J, Yin FCP (2001) Specificity of endothelial cell reorientation in response to cyclic mechanical stretching. J Biomech 34(12):1563-1572

Zielinski A, Linnartz C, Pleschka C, Dreissen G, Springer R, Merkel R, Hoffmann B (2018) Reorientation dynamics and structural interdependencies of actin, microtubules and intermediate filaments upon cyclic stretch application. Cytoskeleton 75:385-394

\section{Preprint}

Chatterjee A, Kondaiah P, Gundiah N, (2019) Stress fiber growth and remodeling determines cellular morphomechanics under uniaxial cyclic stretch, bioRxiv: https://doi.org/10.1101/622092v1. preprint posted 29 April, 2019

Publisher's Note Springer Nature remains neutral with regard to jurisdictional claims in published maps and institutional affiliations. 